\title{
Dissection of complex adult traits in a mouse synthetic population
}

\author{
David T. Burke, ${ }^{1,6}$ Kenneth M. Kozloff, ${ }^{2}$ Shu Chen, ${ }^{3}$ Joshua L. West, ${ }^{1}$ \\ Jodi M. Wilkowski, ${ }^{1}$ Steven A. Goldstein, ${ }^{2}$ Richard A. Miller, ${ }^{3,4,5}$ \\ and Andrzej T. Galecki ${ }^{3}$
}

${ }^{1}$ Department of Human Genetics, University of Michigan, Ann Arbor, Michigan 48109, USA; ${ }^{2}$ Orthopaedic Research Laboratories, Department of Orthopaedic Surgery, University of Michigan, Ann Arbor, Michigan 48109, USA; ${ }^{3}$ Institute of Gerontology and Geriatrics Center, University of Michigan, Ann Arbor, Michigan 48109, USA; ${ }^{4}$ Department of Pathology and Geriatrics Center, University of Michigan, Ann Arbor, Michigan 48109, USA; ${ }^{5}$ Ann Arbor Veterans Administration Medical Center, Ann Arbor, Michigan 48105, USA

\begin{abstract}
Finding the causative genetic variations that underlie complex adult traits is a significant experimental challenge. The unbiased search strategy of genome-wide association (GWAS) has been used extensively in recent human population studies. These efforts, however, typically find only a minor fraction of the genetic loci that are predicted to affect variation. As an experimental model for the analysis of adult polygenic traits, we measured a mouse population for multiple phenotypes and conducted a genome-wide search for effector loci. Complex adult phenotypes, related to body size and bone structure, were measured as component phenotypes, and each subphenotype was associated with a genomic spectrum of candidate effector loci. The strategy successfully detected several loci for the phenotypes, at genome-wide significance, using a single, modestsized population $(N=505)$. The effector loci each explain $2 \%-10 \%$ of the measured trait variation and, taken together, the loci can account for over $25 \%$ of a trait's total population variation. A replicate population $(N=378)$ was used to confirm initially observed loci for one trait (femur length), and, when the two groups were merged, the combined population demonstrated increased power to detect loci. In contrast to human population studies, our mouse genome-wide searches find loci that individually explain a larger fraction of the observed variation. Also, the additive effects of our detected mouse loci more closely match the predicted genetic component of variation. The genetic loci discovered are logical candidates for components of the genetic networks having evolutionary conservation with human biology.
\end{abstract}

Differences among individuals in complex traits are the result of variations in the genome and the interplay of genetic variation with the environment. The genes, variants, and interactions describe the "genetic architecture" of each trait (Flint and Mackay 2009). For human populations, genome-wide association studies (GWAS) provide a robust strategy to search for effector genetic loci and variants, relatively unbiased by prior biological knowledge. In GWAS, genotyped loci that show an association with a measured phenotype are assumed to be physically adjacent to the causal DNA sequences (Hardy and Singleton 2009; Stranger et al. 2011). Nearby transcribed RNAs (or non-coding DNA sequences) are then vetted as candidates for deeper biological analyses using additional human subject resources or experimentation in model organisms (Cantor et al. 2010; Nicolae et al. 2010). Recent genome-wide studies have successfully identified candidates for several complex adult human traits, including type II diabetes, infectious disease susceptibility, serum lipid levels, and cancer (Hindorff et al. 2011).

Three observations are frequently made in human genomewide association studies of genetic architecture. First, individual detected loci-although statistically supported-explain only a small fraction of a trait's observed variation in the population. Second, the number of identified candidates can be large, often exceeding 30 loci for a single phenotype measure. Third, the summed effects of the detected loci are significantly less than the

\section{${ }^{6}$ Corresponding author}

E-mail dtburke@umich.edu

Article published online before print. Article and publication date are at http://www.genome.org/cgi/doi/10.1101/gr.135582.111. total estimated genetic contribution to trait variation (McCarthy et al. 2008; Weiss 2008; Goldstein 2009; Ku et al. 2010). When taken together, these outcomes pose considerable practical challenges to the understanding of the genetic architecture of complex human traits. This is especially true for traits that arise in later adult life and involve complex interactions of genes and alleles with cumulative environmental influences.

The laboratory mouse provides a logical surrogate for analyses of human genetic architecture. The two species maintain evolutionarily conserved biochemistry, tissues, developmental programs, and regulatory systems (Paigen 2003; Quimby et al. 2006). The mouse genome is comparable to the human genome, with a similar estimated number of genes, chromosomal DNA content, and informational complexity (Guan et al. 2008; Guttman et al. 2009; Blake et al. 2011). Importantly, many adult-onset diseases are also broadly similar (Nadon 2006; Rosenthal and Brown 2007). As an exploratory system for understanding complex genetics, the mouse provides significant advantages, including reproducible control over genetic variation and environment (Peters et al. 2007; Beckers et al. 2009). In this study, we examine the genetic basis of phenotypes for body size, growth, and bone properties within a single adult population. Using a simple, low-cost, two-generation breeding system, we have produced a genetically diverse synthetic population of animals derived from well-characterized inbred founder strains. The genome-wide searches within this population successfully identify loci associated with complex genetic architectures of adult traits. Additionally, the genome-wide analyses identify loci associated with phenotype rates of change and measured phenotype subcomponents. In combination with the ex- 
tensive molecular, physiological, genetic, and genomic resources available for the mouse, synthetic population strategies are likely to be useful for the analysis of polygenic phenotypes and may uncover novel candidates for understanding human biology.

\section{Results}

Mouse body size provides an analogy to the extensively studied human trait of adult height. We used mouse size measures to compare the performance of the synthetic population strategy with recent reports from human populations (Soranzo et al. 2009; Allen et al. 2010; Yang et al. 2010). Quantitative values for three measures were obtained from each animal: (1) femur length (right femur, proximal-distal), (2) vertebra length (eighth caudal vertebra, cranio-caudal), and (3) whole body weight (grams, monthly). The individuals in the studied population are progeny of two $F_{1}$ hybrid parents (Fig. 1). Chromosomal recombination in the hybrid parent germlines results in each member of the studied population having a unique genome, with one homolog of each chromosome pair inherited from the $(\mathrm{BALB} / \mathrm{cJ} \times \mathrm{C} 57 \mathrm{BL} / 6 \mathrm{~J}) \mathrm{F}_{1}$ maternal parent and one homolog from the $(\mathrm{C} 3 \mathrm{H} / \mathrm{HeJ} \times \mathrm{DBA} / 2 \mathrm{~J})$ $F_{1}$ paternal parent. The genetically heterogeneous synthetic population has been used to successfully map loci at low resolution for multiple traits, such as T-cell subsets, serum hormone levels, bone properties, and cause of death (Jackson et al. 1999; Harper et al. 2003; Volkman et al. 2003, 2004; Lipman et al. 2004; Reeves et al. 2007). This study uses a high-density full-genome analysis to assess the genetic architecture of complex traits, as a comparison with recent human GWAS population analyses. Each animal in the population $(N=505)$ was genotyped across the genome at loci known to be polymorphic among the four founder strains (558 loci). The measures of body size are consistent with being normally distributed in the synthetic population (Table 1) and have mean values comparable to commonly used inbred strains and $\mathrm{F}_{1}$ hybrids (Grubb et al. 2009).

Genome-wide searches were performed for loci associated with femur length and vertebra length, measured at late adult life (18 mo). Five genomic regions achieve genome-wide significance in each search, $P<0.05$, using a permutation-based statistical

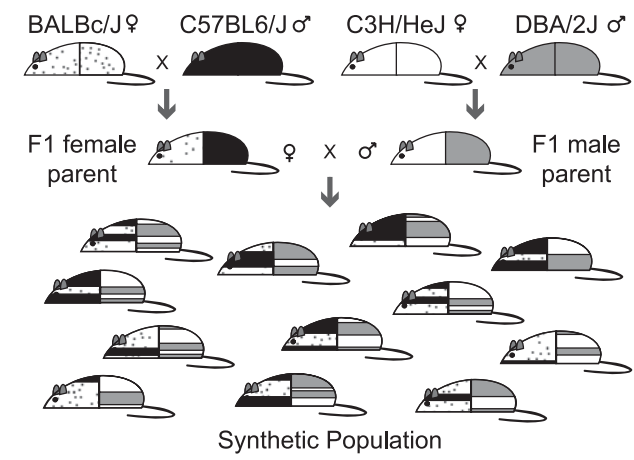

Figure 1. The mouse synthetic population. Each half of a mouse symbol represents one homolog of a chromosome pair. The four founder mice (top row) are inbred strains and are homozygous for all chromosome pairs. The parental mice (second row) are $F_{1}$ hybrids of the founder strains and are heterozygous for every chromosome pair. The progeny of the $\left[F_{1} \times F_{1}\right]$ mating are the genetic equivalent of full siblings and are the studied synthetic population. All synthetic population animals are genotyped across the genome at loci that distinguish the BALB/c) vs. C57BL/6 alleles (maternal-informative loci) or $\mathrm{C} 3 \mathrm{H} / \mathrm{HeJ}$ vs. DBA/2) alleles (paternalinformative loci).
Table 1. Synthetic population adult body size phenotypes

\begin{tabular}{|c|c|c|c|c|c|c|}
\hline Phenotype & Unit & Mean & Median & Std. dev. & Range & $N$ \\
\hline Femur length & $\mathrm{mm}$ & 16.71 & 16.75 & 0.42 & $15.40-17.70$ & 454 \\
\hline $\begin{array}{l}\text { Vertebra length } \\
\text { Body weight: }\end{array}$ & $\mathrm{mm}$ & 4.443 & 4.464 & 0.230 & $3.114-5.202$ & 468 \\
\hline $3 \mathrm{mo}$ & g & 22.6 & 22.3 & 2.7 & $16.3-34.3$ & 416 \\
\hline $12 \mathrm{mo}$ & $\mathrm{g}$ & 30.5 & 30.0 & 4.5 & $20.3-55.1$ & 484 \\
\hline $\begin{array}{l}3 \text { to } 13 \mathrm{mo}, \\
\text { slope }\end{array}$ & $\mathrm{g} / \mathrm{mo}$ & 0.79 & 0.72 & 0.34 & $0.19-2.29$ & 422 \\
\hline
\end{tabular}

model (Table 2). Genotyped loci are denoted by chromosomal location and base-pair distance $(\mathrm{Mb})$ from the centromere (e.g., the locus $\mathrm{CH} 13 \cdot 22$ is on mouse chromosome 13 at $22 \mathrm{Mb}$ ). The individual genomic regions that reach statistical significance in the population nominally explain $3.5 \%-10.8 \%$ of the total population variance, for each phenotype. The genetic architectures of the two traits can be compared directly as genome-wide spectra (Fig. 2A,B), since all phenotype measures and genotypes are obtained from a single laboratory population of animals. Four genomic regions with effects on vertebral length had been noted previously at low genetic resolution; however, the locus on chromosome 13 had not been detected (Reeves et al. 2007).

Using the detected loci, we next examined a simple model of additive effects of the individual loci on bone length. Population members were assigned to groups based on whether they maintained the alleles associated with long femurs ("long" alleles) or the alternative short-femur-associated alleles at the five genomic regions (Table 3). The combined phenotypic effect of the five loci was examined as a retrospective analysis, using a model of the main effects only (ANOVA). The variance explained by summing the five loci for femur length was $27.7 \%$. This simple additive model overfits the data, since the optimal genetic marker loci are chosen from among all tested loci. Nevertheless, the mean difference between the extreme 5-locus groups for femur length is one full millimeter, at a population mean of $16.71 \mathrm{~mm}$. For vertebra length, a similar analysis was performed. The combined additive effect of the "long" alleles at five loci explains $29.0 \%$ of the phenotypic variance in vertebral length, with a mean difference of $0.55 \mathrm{~mm}$, at a population mean length of $4.44 \mathrm{~mm}$. The combined additive effect of five loci is over one-fourth of the total population variance, for each measure.

In human biology, many traits of interest change over the life course. The mouse synthetic population supports time-dependent measures of adult complex traits. To explore this strategy, body weight trajectories for each of the synthetic population animals were examined (Fig. 3). At the population level, the weight distributions are similar to prior observations in ad lib fed laboratory mice (Quimby et al. 2006). Individual animals show considerable variation in their weight trajectories. Four individual animal trajectories are highlighted as examples: the two heaviest and two lightest adult mice in the population at 13 mo. The two lightest animals demonstrated essentially flat growth trajectories, with small early life values and minimal weight increase over time $(\sim 0.2 \mathrm{~g} / \mathrm{mo})$. The single heaviest animal enters the study among the top 5\% of 3-mo-old animals and continues to add weight at a rate far above the population average ( 2.29 vs. $0.79 \mathrm{~g} / \mathrm{mo})$. In contrast, the second-heaviest animal is within the central $50 \%$ of values at $3 \mathrm{mo}$ and then rapidly gains weight $(2.19 \mathrm{~g} / \mathrm{mo})$. As seen in human populations (Clarke et al. 2010), high young-adult weight is positively correlated with rapid weight gain; however,

\section{Genome Research www.genome.org}


Table 2. Loci achieving genome-wide significance for body size

\begin{tabular}{|c|c|c|c|c|c|c|}
\hline Phenotype & $\begin{array}{l}\text { Peak locus } \\
\text { (Chr•Mb) }\end{array}$ & $P$-value ${ }^{a}$ & $\begin{array}{l}\% \text { Variance } \\
\text { explained }^{\mathbf{b}}\end{array}$ & $\begin{array}{l}\text { High value } \\
\text { allele }^{c}\end{array}$ & $\begin{array}{l}\text { Low value } \\
\text { allele }^{c}\end{array}$ & $\begin{array}{l}\text { Genotyped } \\
\text { locus }\end{array}$ \\
\hline \multirow[t]{5}{*}{ Femur length } & $\mathrm{CH} 13 \cdot 22$ & $<0.001$ & 10.2 & B6 & BALB & rs3091203 \\
\hline & $\mathrm{CH} 2 \cdot 157$ & $<0.001$ & 7.1 & $\mathrm{C} 3 \mathrm{H}$ & DBA & rs4223627 \\
\hline & $\mathrm{CH} 9 \cdot 91$ & $<0.001$ & 5.2 & B6 & BALB & D9Mit110 \\
\hline & $\mathrm{CH} 5 \bullet 114$ & $<0.001$ & 5.0 & BALB & B6 & D5Mit25 \\
\hline & $\mathrm{CH} 17 \cdot 17$ & 0.011 & 4.0 & B6 & BALB & rs3657845 \\
\hline \multirow[t]{5}{*}{ Vertebra length } & $\mathrm{CH} 1 \cdot 158$ & $<0.001$ & 10.8 & B6 & BALB & rs4222738 \\
\hline & $\mathrm{CH} 1 \cdot 166$ & $<0.001$ & 8.3 & $\mathrm{C} 3 \mathrm{H}$ & DBA & rs4222769 \\
\hline & $\mathrm{CH} 2 \cdot 111$ & 0.006 & 4.2 & DBA & $\mathrm{C} 3 \mathrm{H}$ & rs 3023543 \\
\hline & $\mathrm{CH} 13 \cdot 16$ & 0.019 & 3.6 & B6 & BALB & rs 13481706 \\
\hline & $\mathrm{CH} 7 \bullet 18$ & 0.026 & 3.5 & DBA & $\mathrm{C} 3 \mathrm{H}$ & D7Mit76 \\
\hline \multirow{3}{*}{$\begin{array}{l}\text { Body weight: } 3 \text { mo. } \\
12 \text { mo. } \\
3-13 \text { mo. slope }\end{array}$} & $\mathrm{CH} 15 \bullet 47$ & 0.005 & 4.8 & BALB & B6 & rs3024123 \\
\hline & $\mathrm{CH} 17 \cdot 17$ & 0.006 & 4.2 & B6 & BALB & rs3657845 \\
\hline & $\mathrm{CH} 2 \cdot 129$ & 0.018 & 3.9 & B6 & BALB & rs 3022902 \\
\hline
\end{tabular}

${ }^{a}$ Genome-wide significance level obtained by permutation-based test of ANOVA model; empirical null distribution of F-test statistic using 1000 permutations.

${ }^{\mathrm{b}}$ Total population variance explained by the single locus at local maxima.

${ }^{C}$ Parental allele associated with the higher or lower phenotype value in the population. (B6) C57BL/6]; (BALB) BALB/CJ; (DBA) DBA/2); (C3H) C3H/HeJ.

${ }^{\mathrm{d}}$ Genotyped locations are either single nucleotide polymorphisms (rs) or simple sequence repeat polymorphisms (D).

3-mo-old animals in the median weight quartile exhibit a range of trajectories, from 0.24 to $1.68 \mathrm{~g} / \mathrm{mo}$.

Genome-wide searches for effector loci were performed using three body weight component measures: (1) early adult weight at 3 mo, (2) late adult weight at $12 \mathrm{mo}$, and (3) the slope of the best-fit linear trajectory for each animal between $3 \mathrm{mo}$ and $13 \mathrm{mo}$ (Fig. 4). In each search, one chromosomal region was identified having genome-wide statistical significance of $P<0.05$. Each significant chromosomal region was associated with a phenotypic effect that explains $>3 \%$ of the total population variance (Table 2 ). Early adult weight is associated with a locus on chromosome 15 , late adult weight with a locus on chromosome 17 , and weight-gain rate with a locus on chromosome 2 . The cumulative percent of the phenotypic variance explained by an additive model for the three loci on late adult weight was $10.2 \%$. Late adult weight is dependent on an animal's early weight and its weight trajectory. Closer examination of the 12-mo weight spectrum (Fig. 4, bottom) shows a "shadow effect" of associations that map to the $\mathrm{CH} 15 \bullet 47$ and $\mathrm{CH} 2 \bullet 129$ regions, originally detected for 3-mo weight and weight trajectory, respectively. Animals maintaining the high-weight alleles at $\mathrm{CH} 2$, $\mathrm{CH} 15$, and $\mathrm{CH} 17$, in comparison with animals with three lowerweight alleles, have a mean difference in late adult weight of $>4 \mathrm{~g}$, at a population mean of $30.5 \mathrm{~g}$. In a separate mouse population, a locus on $\mathrm{CH} 15$ was associated with early life body weight; consequently, the current observation likely confirms this result (Miller et al. 2002).

We next examined the genetic architecture of three interrelated measures involved in adult bone phenotypes. For each mouse, microcomputed tomography was used to determine trabecular bone morphology and microstructure, at 18 mo. Quantitative measures of bone volume fraction (bone volume/total volume) and trabecular organization (plate number/millimeter) were taken for the eighth caudal vertebra. The same bone was also subjected to mechanical compression to obtain the ultimate load to failure (Reeves et al. 2007). Genome-wide searches for loci associated with each measure were then performed (Table 4). Similar to the results for body size, the genomic architecture of bone uncovers multiple loci, with a complex interrelationship between genetic effectors and phenotype measurements. Again, the genetic architecture spectra of the traits can be compared directly since all phenotypes were obtained from a single population (Fig. 5).

To test the reproducibility of the genome-wide search strategy, a second population of progeny from a $(\mathrm{BALB} / \mathrm{cJ} \times$ C57BL/6J) $\mathrm{F}_{1}$ maternal parent by $(\mathrm{C} 3 \mathrm{H} /$ HeJ $\times$ DBA/2J) $F_{1}$ paternal parent cross was available for examination $(N=378)$. The four founder strains are maintained by strict inbreeding by the Jackson Laboratories; consequently, the underlying genetic differences between the two populations are likely to be minimal even though they were produced $>3 \mathrm{yr}$ apart. Each animal in the replicate population was genotyped across the genome and measured in vivo for adult femur length at $15 \mathrm{mo}$. The five maximal loci identified in the initial population search (Table 2) were independently tested for confirmation. For all five chromosomal regions, a genotyped locus within $15 \mathrm{Mb}$ of the initially determined maximal position yielded a statistically significant pointwise effect: CH2•157, $P<0.001 ;$ CH5•114, $P=0.02$; CH9•91 $P<0.001$; $\mathrm{CH} 13 \cdot 22, P<0.001$; and $\mathrm{CH} 17 \cdot 17, P=0.002$. Although the replication population was smaller ( 378 vs. 454$)$, it was able to confirm each of the major effector loci for femur length. The observed inflation of phenotype effects during an initial search (the "winners curse") is a predicted phenomenon in genome-wide analyses (Lohmueller et al. 2003; Kraft 2008).

When combined, the two populations provided a total of 832 animals derived from $(\mathrm{BALB} / \mathrm{cJ} \times \mathrm{C} 57 \mathrm{BL} / 6 \mathrm{~J}) \mathrm{F}_{1}$ by $(\mathrm{C} 3 \mathrm{H} / \mathrm{HeJ} \times$ DBA/2J) $F_{1}$ parents and having a phenotype measurement for adult femur length. We performed a new genome-wide search on the merged data to assess the impact of total population size on the ability to detect effector loci. In the expanded population, 11 genomic regions achieved genome-wide statistical significance (Table 5). The individual genomic regions nominally explain $1.7 \%-6.6 \%$ of the population variance (Fig. 6). Taken together, the population phenotypic variance explained by summing the 11 candidate effector loci was 30.5\% (ANOVA; modeled using main effects only). As anticipated, both the number of significant loci detected and the explained cumulative effect of the loci are increased in the larger population.

\section{Discussion}

Our experimental results show that a synthetic mouse population can provide a simple and reproducible strategy for dissecting the complex genomic architectures of multiple adult mammalian traits. As in human population studies, several loci for each phenotype measure can be obtained using the synthetic population and a high-resolution genome-wide search. However, two important quantitative differences are clear. First, in the mouse population, each detected locus may impart a substantial biological effect on the phenotype. The individual loci contribute $\sim 2 \%-10 \%$ of the total population variance. As a comparison, a recent human population meta-analysis of adult height observed no single locus with an effect $>0.5 \%$ of the total variance, in a population of 183,727 

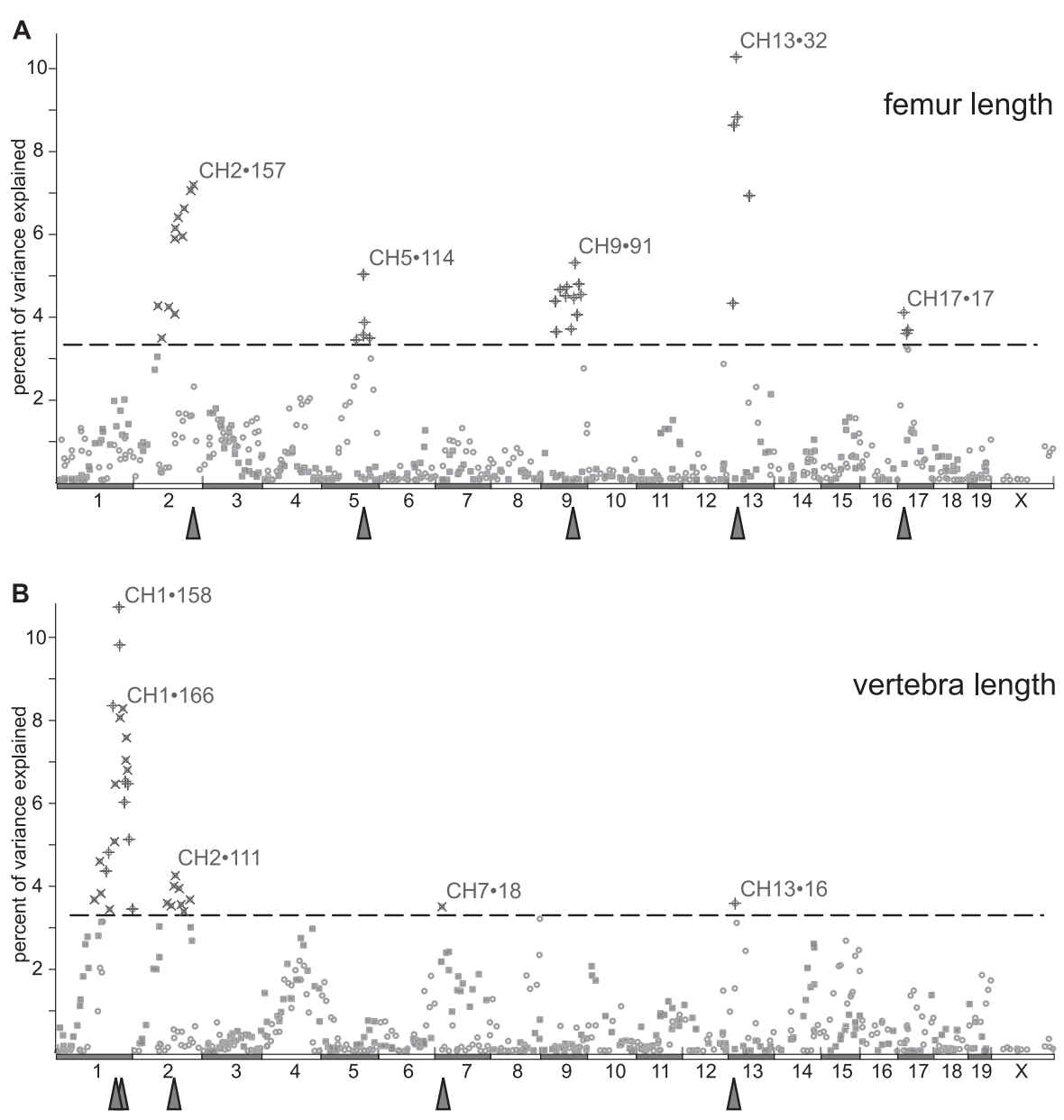

Figure 2. Genome-wide analysis of femur and vertebra length. For each genotyped locus, the display shows the percent of the total measured population variance explained $\left(R^{2} \times 100\right)$. (Horizontal dashed line) The genome-wide significance level of $P<0.05$. (Crossed symbols) The individual loci reaching significance. In each statistically significant genomic region, the locus with maximal effect is labeled with the chromosome number and distance from the centromere, in megabase pairs (Mb, triangle). (A) Loci associated with right femur length $(N=454)$. (B) Loci associated with caudal vertebra (C8) length $(N=468)$. In both panels, (circles) maternal-informative loci (BALB/cJ vs. C57BL/6J alleles); (squares) paternal-informative loci ( $\mathrm{C} 3 \mathrm{H} / \mathrm{HeJ}$ vs. DBA/2J alleles).

individuals (Yang et al. 2010). The "signal-to-noise" ratio of each mouse locus, in the context of a population of heterogeneous genomes, is large. Second, the additive effects of the detected loci in the mouse population more closely approximate the total expected genetic variation for complex traits. The estimated genetic contribution to body weight and femur length is $60 \%-80 \%$, based on mouse strain intercross studies (Reed et al. 2007; Jepsen et al. 2009). Using our merged population of 832 individuals, the additive effect of the 11 significant loci for femur length explained $30 \%$ of the total variation (or over $60 \%$ of the expected genetic variation). In contrast, the additive effect of 180 detected loci involved in human height was able to explain $<12 \%$ of the population variance, in a population more than 200 times larger (Soranzo et al. 2009). This difference in genetic architecture may be, in part, a result of the mouse synthetic population being limited to a maximum of four alleles at any locus, as well as by the ancestral relatedness of the inbred founder strains (Beck et al. 2000). Additionally, the gene variants in the synthetic population are limited to those present in the inbred lines. It is possible that many of these variants are rare in wild mouse populations (or are new mutations arising after isolation of the strains from the wild). The rare alleles are fixed during the inbreeding of the parental lines and now appear at high frequency in the synthetic population. Human population variants that contribute to genome-wide search outcomes, in contrast, are typically common in the human population, and these common variants appear to have smaller effect sizes than rare alleles. Importantly, the reduced complexity of the synthetic population genetic architecture will likely simplify the experimental challenges of subsequent hypothesis-based testing of gene candidates and gene-by-gene interaction effects.

Mouse synthetic populations also demonstrate qualitative advantages for exploring genetic architecture. Paramount among these is the experimental replication of highly heterogeneous populations. Synthetic laboratory populations, such as the one described here, are easily produced from available inbred founders. Individuals in the replicate populations are not genetically identical (Fig. 1), yet the population-level genotype and phenotype characteristics remain consistent. As demonstrated for femur length, replicate populations can confirm genomewide effector loci, increase the sensitivity of locus detection, and reproduce the evidence for causation. All of the animals in the present study were derived from a single mating strategy and held in a single vivarium environment; consequently, population replication should allow the examination of alternative genomic sources (e.g., other inbred lines, or inbred lines maintaining transgenes or mutations) or alternative environments (e.g., modified diets or administered drugs) on genetic architecture. The number of animals entered into a new exploratory population can be modest during a preliminary analysis and then expanded subsequently.

The founder strains were not selected based on phenotype, but to provide genotypic variation in the population. As a consequence, the single population displays gene-based variation for many measures simultaneously. Observation of multiple phenotypes within a single population allows direct comparisons of genetic architecture across interrelated physiological domains (Kenney-Hunt et al. 2008). In this population, submeasures (or endophenotypes) of a trait can yield distinct spectra of effector loci. We detected some loci associated with one body size measure, for example, distal CH5 with femur length. Other loci, however, may be identifying common regulators of several components of size, such as proximal CH13 (femur length and vertebra length) and proximal $\mathrm{CH} 17$ (femur length and body weight). Similarly, the genetic loci associated with the mechanical performance of vertebral bone (measured as ultimate load to failure) are not a simple

\section{Genome Research www.genome.org}


Table 3. Additive effect of loci associated with femur length and vertebra length

\begin{tabular}{lcccccr}
\hline Phenotype $^{\mathbf{a}}$ & $\begin{array}{c}\text { Combined "long" } \\
\text { alleles }\end{array}$ & Mean & Median & Std. dev. & Range & $\boldsymbol{N}^{\mathbf{c}}$ \\
\hline \multirow{2}{*}{ Femur length } & 5 long & $\mathbf{1 7 . 0 8}$ & 17.15 & 0.29 & $16.40-17.57$ & 19 \\
& 4 long & 17.01 & 17.05 & 0.32 & $16.18-17.58$ & 63 \\
& 3 long & 16.78 & 16.77 & 0.34 & $16.00-17.70$ & 140 \\
& 2 long & 16.66 & 16.69 & 0.37 & $15.57-17.40$ & 122 \\
& 1 long & 16.48 & 16.52 & 0.38 & $15.70-17.32$ & 68 \\
Vertebra length & 0 long & $\mathbf{1 6 . 0 7}$ & 15.97 & 0.41 & $15.40-16.95$ & 21 \\
& 5 long & $\mathbf{4 . 6 8 5}$ & 4.626 & 0.230 & $4.374-5.202$ & 15 \\
& 4 long & 4.602 & 4.644 & 0.179 & $4.014-5.022$ & 69 \\
& 3 long & 4.494 & 4.500 & 0.152 & $4.086-4.806$ & 158 \\
& 2 long & 4.393 & 4.428 & 0.239 & $3.114-4.932$ & 135 \\
& 1 long & 4.295 & 4.302 & 0.187 & $3.654-4.806$ & 69 \\
& 0 long & $\mathbf{4 . 1 3 3}$ & 4.194 & 0.296 & $3.240-4.518$ & 18 \\
\hline
\end{tabular}

Bolded values indicate the full additive effect of the five loci, for each phenotype.

${ }^{\mathrm{a}}$ All measures are in millimeters.

b Loci and alleles associated with long or short phenotype groups are given in Table 2.

cNumber of genotyped individuals in the group.

reflection of the loci influencing overall bone density or structural organization of the trabecular network. While the locus on distal CH1 may have a common impact on both trabecular morphology and mechanical strength, the locus at proximal $\mathrm{CH} 17$ identifies an effector with its largest impact on mechanical strength. In a previous analysis (Reeves et al. 2007), a CH17 locus was associated with vertebral cross-sectional area at proximal, mid-length, and distal locations; consequently, it is plausible that this may account for effects on the load to failure.

Genome-wide scans of the synthetic population are readily adaptable to a range of analytical demands, since sequence variations among the inbred strains are known across the genome (Frazer et al. 2007). As described in previous reports, low-resolution scans (i.e., 185 genotyped loci per genome) can find broad localizations of candidate loci (Jackson et al. 1999; Harper et al. 2003; Volkman et al. 2003, 2004; Lipman et al. 2004). Low-resolution scans, however, can fail to detect loci with modest phenotype effect or loci that are genetically distant from typed markers. For example, preliminary genotyping yielded four chromosomal regions associated with bone volume fraction, two regions associated with ultimate load to failure, and four regions associated with trabecular plate number, as statistically significant at genome-wide $P<0.05$ (Reeves et al. 2007). The higher-resolution genotyping described here reveals an additional $\mathrm{X}$-linked region modulating bone volume fraction and three additional loci associated with trabecular plate number $(\mathrm{CH} 7, \mathrm{CHX}$, and maternal-derived $\mathrm{CH} 14)$ at genome-wide $P<0.05$. Permutationbased determination of genome-wide significance adjusts for the number of tested genotype loci to match the scan marker density. Increasing the number of animals in the population and the density of genotyped loci can refine candidate locus intervals for all measured phenotypes, simultaneously.

Although we have tested a single breeding structure at a modest population size, the experiments uncover a rich complexity of genetic effectors on adult mammalian phenotypes. Further experience with mouse synthetic populations will be required to assess several important issues, including the impact of (1) sampling wider genetic variation using alternative founder strains; (2) non-additive or epistatic interactions among genes and alleles (Hanlon et al. 2006); and (3) interactions between genetic architecture and the environment. The identification of the causative DNA sequence associated with each effector locus peak will be essential for com- plete analysis. Chromosomal locations of individual effector genes may be refined using established experimental strategies and knowledge of the founder-strain genomic sequences. For example, founderstrain crosses can be used to segregate and localize genes, even when a locus is not the sole determiner of the phenotype (Flint et al. 2006). Alternatively, strainspecific alleles and haplotypes can be introduced onto uniform strain backgrounds by transgenesis, using large DNA segments as bacterial artificial chromosomes (Antoch et al. 1997), or by breeding of consomic strains (Takada et al. 2008). Importantly, candidate alleles discovered in the synthetic population can be confirmed by testing within a homologous (mouse) experimental system.

Mouse population strategies, in general, should prove to be broadly useful for genetic architecture studies (Valdar et al. 2006a,b; Chesler et al. 2008; Wagner et al. 2008). In the $(\mathrm{BALB} / \mathrm{cJ} \times \mathrm{C} 57 \mathrm{BL} / 6 \mathrm{~J}) \mathrm{F}_{1} \times(\mathrm{C} 3 \mathrm{H} / \mathrm{HeJ} \times$ $\mathrm{DBA} / 2 \mathrm{~J}) \mathrm{F}_{1}$ cross, the parental animals are highly fertile, easily obtained, and have extensive genome sequence available. Prior studies using genetically heterogeneous $\left[\mathrm{F}_{1} \times \mathrm{F}_{1}\right]$ cross-strategies support a range of successful applications to understanding quantitative phenotypes, particularly for evaluation of age-dependent change. Assessments of serum hormone levels, weight trajectories,

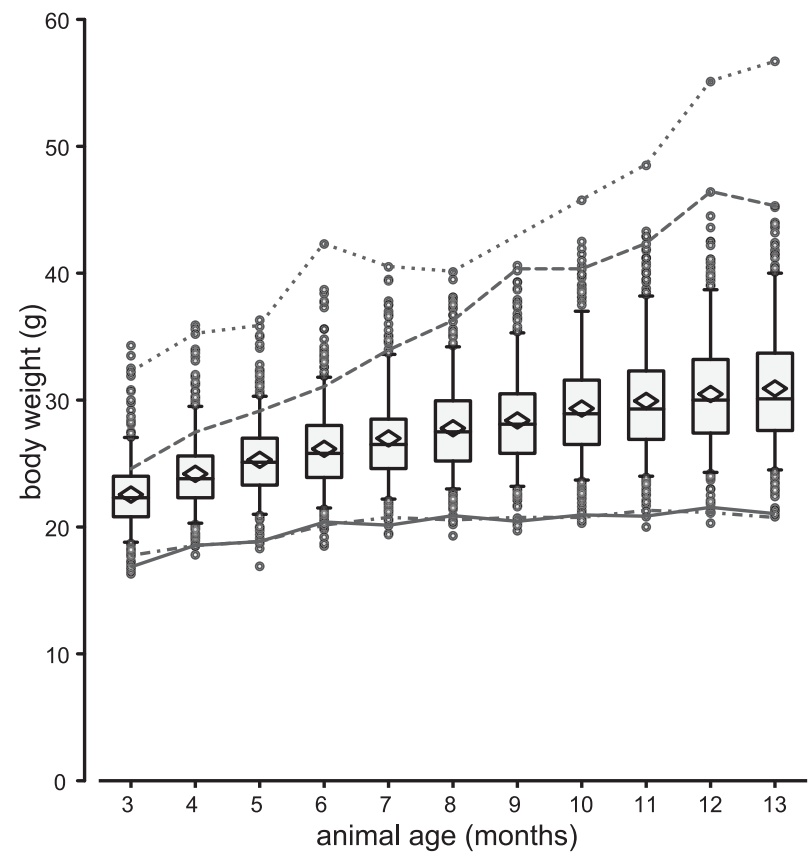

Figure 3. Time course of synthetic population body weight. Longitudinal body weight distributions of the synthetic population from ages 3 13 mo $(\min N=416 ; \max N=490)$. At each month, (open box) the interquartile range; (whiskers) the $5 \%-95 \%$ range; and (open circles) the individual values of the upper and lower $5 \%$. The longitudinal trajectories for the two highest-weight individuals ([dotted line] animal P0358, $2.29 \mathrm{~g} / \mathrm{mo}$; [single dashed line] animal P1023, $2.19 \mathrm{~g} / \mathrm{mo}$ ) and the two lowest-weight individuals are shown ([double-dashed line] animal P1373, $0.19 \mathrm{~g} / \mathrm{mo}$; [solid line] animal P5909, $0.19 \mathrm{~g} / \mathrm{mo})$. 


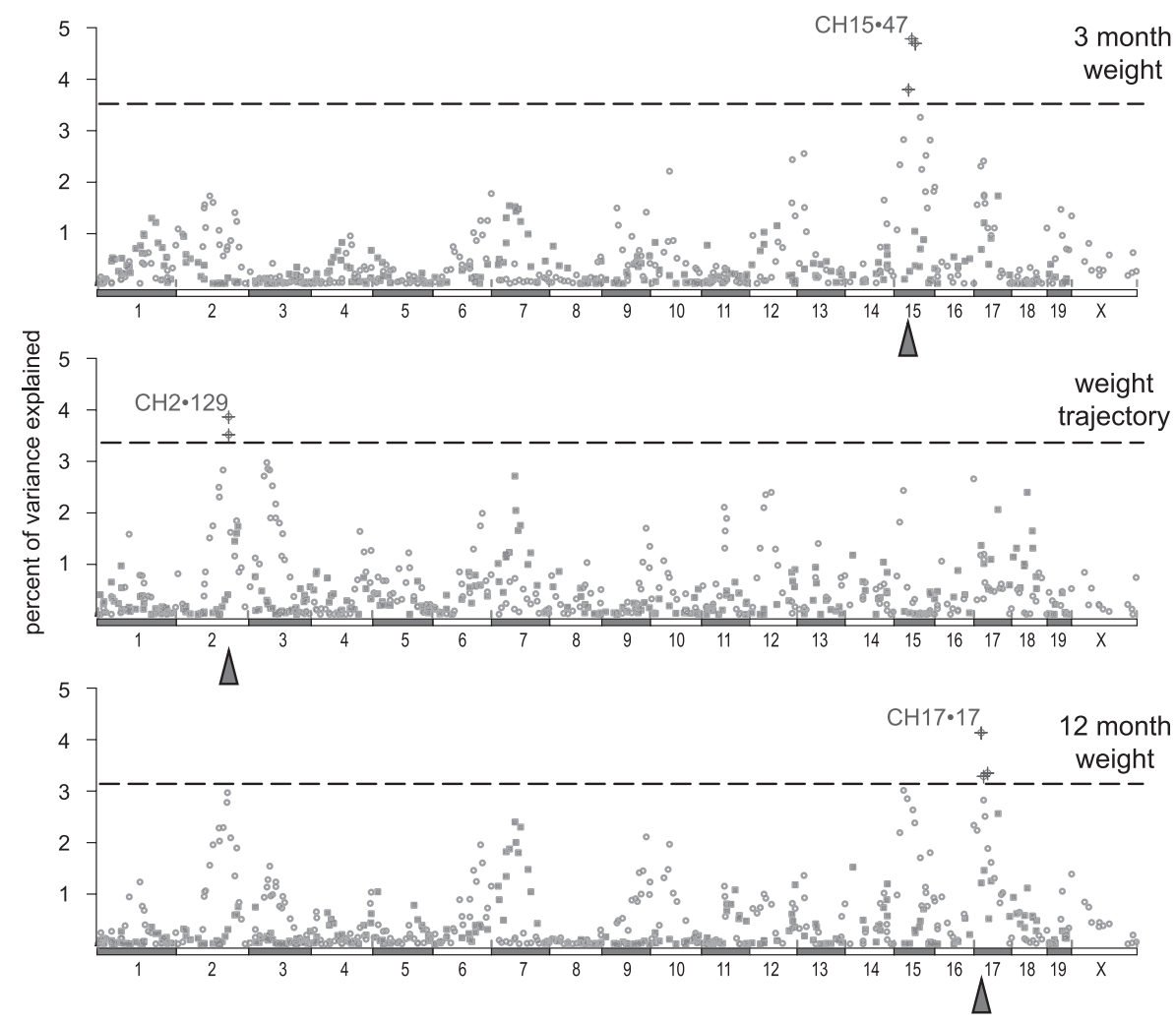

Figure 4. Genome-wide analysis of adult body weight. Genome-wide association plots of 3-mo weight, adult weight trajectory (3- to 13-mo slope), and 12-mo weight. (Horizontal dashed line) The genome-wide significance level of $P<0.05$. (Crossed symbols) The individual loci reaching significance; (triangles) the regional maxima for each measure. (Circles) Maternal-informative loci (BALB/cJ vs. C57BL/6J alleles); (squares) paternal-informative loci (C3H/HeJ vs. DBA/2J alleles).

bone properties, and terminal pathology, as well as interactive effects involving three-way and four-way epistatic associations on serum hormone levels, have revealed additional complexity in the genetic architecture within synthetic populations (Hanlon et al. 2006).

The studied synthetic population animals maintain fully informative, linkage phase-known haplotypes and have not undergone selection by inbreeding depression. As with human populations (and wild populations of mice), the synthetic population members are heterozygous for many loci. Consequently, the phenotypic impact of allelic variation at a gene occurs in the context of a robust, hybrid genome (Phelan and Austad 1994). Relative to human populations, the heterologous mouse studies require considerably fewer resources to explore complex adult traits. It is unlikely that identical alleles are segregating in humans and mice; however, the syntenic genes discovered in the mouse are strong candidates for components of evolutionarily conserved genetic networks. Once identified, specific "candidate gene" hypotheses formulated from synthetic mouse populations may be tested in human populations. The evolutionary linkage between mammalian species supports their use as parallel strategies toward understanding the genetic sources of phenotype variation.

\section{Methods}

Mouse breeding and husbandry

All parental animals, $(\mathrm{BALB} / \mathrm{cJ} \times \mathrm{C} 57 \mathrm{BL} / 6 \mathrm{~J}) \mathrm{F}_{1}$ maternal parent and $(\mathrm{C} 3 \mathrm{H} / \mathrm{HeJ} \times \mathrm{DBA} / 2 \mathrm{~J}) \mathrm{F}_{1}$ paternal parent, were obtained from the
Jackson Laboratories (stock numbers 100007 and 100004, respectively; Bar Harbor, ME). The initial population of 505 female animals was bred from these parents and maintained in specific pathogen-free rooms under identical environmental conditions (12:12 h light: dark cycle, $23^{\circ} \mathrm{C}$, four females per cage). Mice were given ad libitum access to water and laboratory mouse chow. Sentinel mice were tested every 3 mo to verify the pathogen-free status, and all tests were negative throughout the study. Each animal in the initial population was sacrificed at 18 mo of age and dissected (Volkman et al. 2004). The replicate population of 378 females was bred using the same $F_{1}$ strains as parents, and the members of the replicate population were held in the pathogen-free facilities under identical conditions. All of the animal experimentation was performed under the appropriate guidelines, as stipulated by the University of Michigan Medical School.

\section{Genotype analysis}

For all animals, genomic DNA was prepared from $1-\mathrm{cm}$ sections of tail obtained at 3 mo of age. Genomic DNA was genotyped using either ligation-detection reactions or fluorescent-labeled PCR, followed by gel electrophoresis. In the initial population of 505 animals, 558 biallelic informative loci were examined across the genome, with 311 loci informative for the maternal-derived and 247 loci informative for paternal-derived chromosomes. Each genotyped locus is identified by its chromosome and the genome sequence distance from the centromere (Hanlon et al. 2006). The

Table 4. Loci achieving genome-wide significance for vertebra structure

\begin{tabular}{|c|c|c|c|c|}
\hline Phenotype & $\begin{array}{l}\text { Peak locus } \\
\text { (Chr•Mb) }\end{array}$ & $P$-value ${ }^{a}$ & $\begin{array}{l}\text { \% Variance } \\
\text { explained }^{\mathrm{b}}\end{array}$ & $\begin{array}{l}\text { Genotyped } \\
\text { locus }^{\mathrm{c}}\end{array}$ \\
\hline \multirow[t]{5}{*}{ Bone volume fraction } & $\mathrm{CH} 14 \cdot 86$ & $<0.001$ & 9.4 & rs31204804 \\
\hline & $\mathrm{CH} 5 \cdot 114$ & $<0.001$ & 6.9 & D5Mit25 \\
\hline & $\mathrm{CH} 8 \cdot 118$ & $<0.001$ & 5.5 & rs 13480005 \\
\hline & $\mathrm{CHX} \cdot 94$ & 0.001 & 4.7 & rs3023499 \\
\hline & $\mathrm{CH} 14 \cdot 79$ & 0.002 & 4.7 & rs 3665356 \\
\hline \multirow{6}{*}{$\begin{array}{l}\text { Trabecular number } \\
\text { density }\end{array}$} & $\mathrm{CH} 14 \cdot 84$ & $<0.001$ & 6.4 & rs13482281 \\
\hline & $\mathrm{CH} 8 \cdot 118$ & $<0.001$ & 4.9 & rs13480005 \\
\hline & $\mathrm{CH} 1 \cdot 145$ & $<0.001$ & 4.2 & rs 4222673 \\
\hline & $\mathrm{CH} 7 \bullet 89$ & 0.009 & 4.1 & rs 13479393 \\
\hline & $\mathrm{CH} 14 \cdot 98$ & 0.022 & 3.5 & rs 13482334 \\
\hline & $\mathrm{CHX} \cdot 141$ & 0.023 & 3.4 & rs13480477 \\
\hline \multirow[t]{2}{*}{ Mechanical strength } & $\mathrm{CH} 1 \cdot 145$ & 0.011 & 3.9 & rs13476148 \\
\hline & $\mathrm{CH} 17 \cdot 25$ & 0.019 & 3.7 & D17Mit46 \\
\hline
\end{tabular}

${ }^{a}$ Genome-wide significance level obtained by permutation-based test of ANOVA model; empirical null distribution of F-test statistic using 1000 permutations.

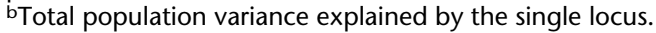

${ }^{\mathrm{C}} \mathrm{Genotyped}$ locations are either single nucleotide polymorphisms (rs) or simple sequence repeat polymorphisms (D).

\section{Genome Research www.genome.org}




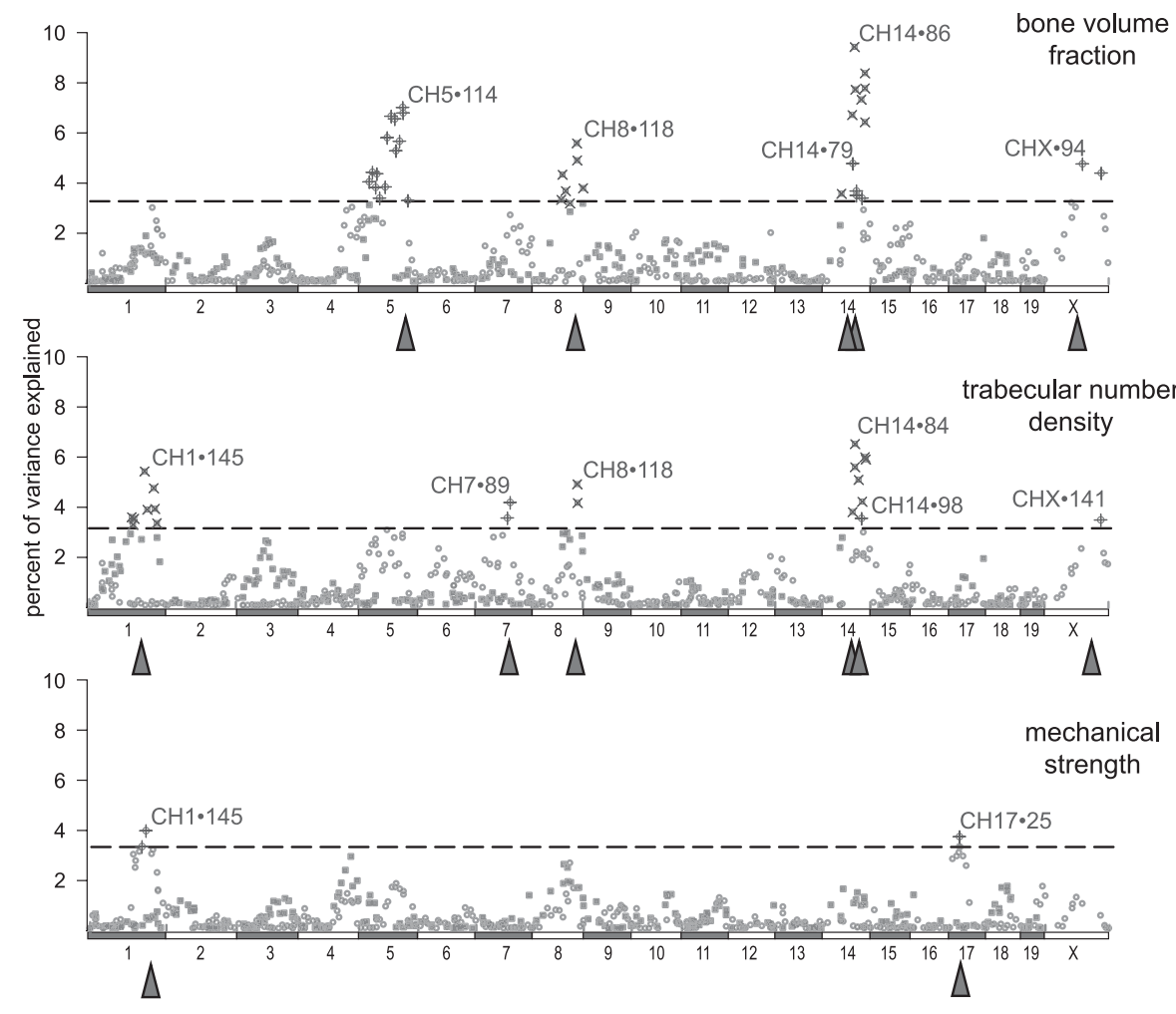

Figure 5. Genome-wide analysis of bone as component measures. Genome-wide association plots for vertebral bone volume fraction (top, $N=472$ ), trabecular plate number density (middle, $N=472$ ), and mechanical strength (bottom; ultimate load to failure, $N=451$ ). (Horizontal dashed line) The genome-wide significance level of $P<0.05$. (Crossed symbols) The individual loci reaching significance; (triangles) the regional maxima for each measure.

median distance between adjacent informative markers is 7.6 $\mathrm{Mb}$. The replicate population of 378 animals was genotyped at a subset of the loci (356 of the 558 loci).

\section{Phenotype analysis}

Synthetic population animals were entered into the study in a staggered fashion, at a rate of 25-35 mice per month. Each animal was weighed to the nearest $0.1 \mathrm{~g}$ at monthly intervals from 3 mo of age until death or sacrifice. The rate of change in body weight was estimated for animals having at least seven weight values measured between $3 \mathrm{mo}$ and $13 \mathrm{mo}$, using the slope of the linear regression line of the values. Animals in the initial population $(N=505)$ were euthanized at 18 mo of age, at which time right femur and eighth caudal vertebrae were removed, dissected free of soft tissue, and frozen in phosphate-buffered saline solution. Bones were scanned on either a custom-built cone beam 3D microcomputed tomography system, as previously described and validated (Feldkamp et al. 1989; Kuhn et al. 1990), or using a commercially available micro-CT system (GE Healthcare Biosciences). Femoral scans were reconstructed at a voxel size of $25 \times 25 \times 25 \mu^{3}$, and caudal vertebra were reconstructed with $18 \times 18 \times 18 \mu \mathrm{m}^{3}$ voxel size to generate three-dimensional digitized images (MicroView software, GE Healthcare Biosciences). Femur lengths and vertebra lengths were obtained from the images. Standard trabecular stereology parameters $P p$ and $P_{L}$ were calculated from the digitized images. Bone volume fraction was calculated as occupied bone volume divided by total bone volume (parameter $P p$; the number of voxels greater than threshold divided by the total number of voxels in the volume). Trabecular plate number was calculated as the number of intersections of test lines with the bone-to-marrow interface, divided by the total line length (in millimeters), within the same volume image (parameter $P_{L}$ ). The mechanical strength of each vertebra was measured by loading to failure in compression, using a servohydraulic testing instrument generating a constant displacement rate between parallel rigid loading platens (858 Mini-Bionix II; MTS Systems). Micro-CT scans of femur length for the replicate population members $(N=378)$ were obtained in vivo at 15 mo of age.

\section{Genome-wide genetic locus search}

A single-point genome-wide search was performed for each trait, as previously described (Jackson et al. 1999; Harper et al. 2003). Briefly, one-way analysis of variance models, with one trait as the dependent variable and each biallelic marker as the factor with two levels, was used for all 558 genotyped loci. The strength of associations between genetic markers and traits was evaluated using a permutation-based test of statistical significance. This test generates an experiment-wide acceptance criterion to take into account multiple hypotheses and to avoid type I error inflation. A null distribution was generated based on 1000 shuffles of the original phenotype data, for each examined phenotype (empirical null distribution, F-test statistic). Phenotype values were permuted, holding the genome of each animal constant. The additive phenotypic effects of multiple loci were examined using a statistical model of the main effects only. The effect size of each locus was assessed using analysis of variance and linear regression analysis. Replication results on the second pop-

Table 5. Loci achieving genome-wide significance for femur length in combined population

\begin{tabular}{|c|c|c|c|c|}
\hline $\begin{array}{l}\text { Peak locus } \\
(\text { Chr•Mb) }\end{array}$ & $\begin{array}{l}\text { Replicated in } \\
\text { 2nd population }\end{array}$ & $P$-value ${ }^{b}$ & $\begin{array}{l}\% \text { Variance } \\
\text { explained }^{\mathrm{c}}\end{array}$ & $\begin{array}{l}\text { Genotyped } \\
\text { locus }\end{array}$ \\
\hline $\mathrm{CH} 2 \cdot 131$ & $\checkmark$ & $<0.001$ & 6.7 & rs4223505 \\
\hline $\mathrm{CH} 13 \cdot 22$ & 1 & $<0.001$ & 6.5 & rs3091203 \\
\hline $\mathrm{CH} 9 \bullet 86$ & $\checkmark$ & $<0.001$ & 4.0 & rs4227806 \\
\hline $\mathrm{CH} 17 \cdot 17$ & $\checkmark$ & $<0.001$ & 3.8 & rs3657845 \\
\hline $\mathrm{CH} 2 \cdot 159$ & & $<0.001$ & 2.9 & rs13476874 \\
\hline $\mathrm{CH} 3 \cdot 50$ & & $<0.001$ & 2.8 & rs3022957 \\
\hline $\mathrm{CH} 5 \bullet 105$ & $\checkmark$ & 0.003 & 2.1 & rs6403048 \\
\hline $\mathrm{CH} 1 \bullet 114$ & & 0.005 & 2.0 & rs13476056 \\
\hline $\mathrm{CH} 13 \cdot 105$ & & 0.010 & 1.9 & rs6389588 \\
\hline $\mathrm{CH} 3 \cdot 135$ & & 0.019 & 1.8 & rs 13477400 \\
\hline $\mathrm{CH} \cdot \bullet 11$ & & 0.042 & 1.7 & rs13480069 \\
\hline
\end{tabular}

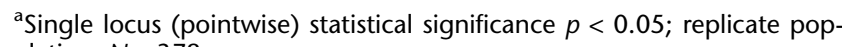
ulation, $N=378$.

${ }^{\mathrm{b}}$ Genome-wide significance level obtained by permutation-based test of ANOVA model; empirical null distribution of F-test statistic using 1000 permutations; $N=832$.

'Total population variance explained by the single locus.

${ }^{\mathrm{d}}$ Genotyped locations are single nucleotide polymorphisms (rs). 


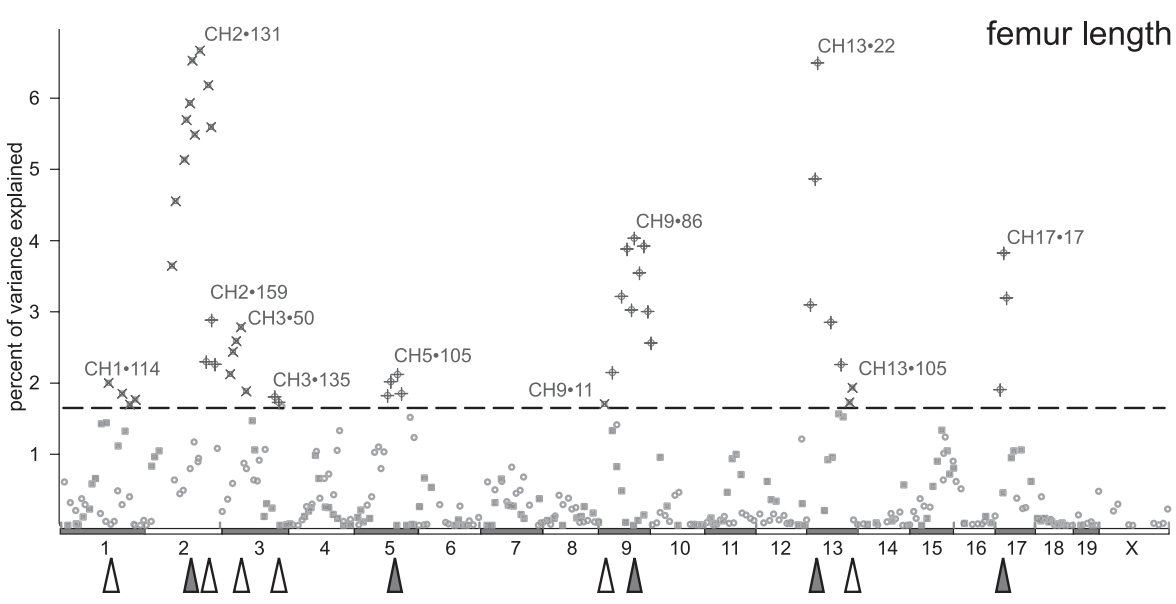

Figure 6. Analysis of combined synthetic populations for femur length. Femur length measures, derived from two separate experimental cohorts, analyzed as a single combined population ( $N=832$; 454 initial population, 378 replicate population). (Horizontal dashed line) The genome-wide significance level of $P<0.05$. (Crossed symbols) The individual loci reaching significance; (open triangles) the genomic locations of loci identified in the initial synthetic population; (filled triangles) loci identified in the combined analysis.

ulation were tested for each locus, independently, using a $t$-test. All tests were performed using SAS software (v9-1, SAS Institute Inc.).

\section{Data access}

The synthetic population genotype and phenotype data used for this analysis can be found online at the QTL Archive, curated by the Jackson Laboratories. The web location is http://qtlarchive. org/db/q?pg=projdetails\&proj=burke_2012.

\section{Acknowledgments}

We thank Matt Zawistowski, Julie Douglas, Jun Li, and Jeff Long for useful discussions and constructive criticism of the manuscript. We thank Lisa Burmeister, Maggie Lauderdale, Sabrina Friedline, Grant Reeves, Suzanne Volkman, and Barbara McCreadie for scientific assistance.

Author contributions: D.T.B., A.T.G., K.M.K., S.A.G., and R.A.M. designed the experiments, collected data, and wrote the manuscript. S.C. and A.T.G. designed the statistical analysis strategy and performed statistical analyses. J.L.W. and J.M.W. developed the genotyping strategy and performed genotyping.

\section{References}

Allen HL, Estrada K, Lettre G, Berndt SI, Weedon MN, Rivadeneira F, Willer CJ, Jackson AU, Vedantam S, Raychaudhuri S, et al. 2010. Hundreds of variants clustered in genomic loci and biological pathways affect human height. Nature 467: 832-838.

Antoch MP, Song EJ, Chang AM, Vitaterna MH, Zhao Y, Wilsbacher LD, Sangoram AM, King DP, Pinto LH, Takahashi JS. 1997. Functional identification of the mouse circadian clock gene by transgenic BAC rescue. Cell 89: 655-667.

Beck JA, Lloyd S, Hafezparast M, Lennon-Pierce M, Eppig JT, Festing MF, Fisher EM. 2000. Genealogies of mouse inbred strains. Nat Genet 24: 23-25.

Beckers J, Wurst W, De Angelis MH. 2009. Towards better mouse models: Enhanced genotypes, systemic phenotyping and envirotype modeling. Nat Rev Genet 10: $371-380$.

Blake JA, Bult CJ, Kadin JA, Richardson JE, Eppig JT, The Mouse Genome Database Group. 2011. The Mouse Genome Database (MGD): Premier model organism resource for mammalian genomics and genetics. Nucleic Acids Res 39: D842-D848.
Cantor RM, Lange K, Sinsheimer JS. 2010. Prioritizing GWAS results: A review of statistical methods and recommendations for their application. Am J Hum Genet 86: 622.

Chesler EJ, Miller DR, Branstetter LR, Galloway LD, Jackson BL, Philip VM, Voy BH, Culiat CT, Threadgill DW, Williams RW, et al. 2008. The Collaborative Cross at the Oak Ridge National Laboratory: Developing a powerful resource for systems genetics. Mamm Genome 19: 382-389.

Clarke PJ, O'Malley PM, Schulenberg JE, Johnston LD. 2010. Midlife health and socioeconomic consequences of persistent overweight across early adulthood: Findings from a national survey of American adults (1986-2008). Am J Epidemiol 172: 540-548.

Feldkamp LA, Goldstein SA, Parfitt AM, Jesion G, Kleerekoper M. 1989. The direct examination of three-dimensional bone architecture in vitro by computed tomography. J Bone Miner 4: 3-11.

Flint J, Mackay TFC. 2009. Genetic architecture of quantitative traits in mice, flies, and humans. Genome Res 19: 723-733.

Flint J, Valdar W, Shifman S, Mott R. 2006. Strategies for mapping and cloning quantitative trait genes in rodents. Nat Rev Genet 6: 271-286.

Frazer KA, Eskin E, Kang HM, Bogue MA, Hinds DA, Beilharz EJ, Gupta RV Montgomery J, Morenzoni MM, Nilsen GB, et al. 2007. A sequencebased variation map of 827 million SNPs in inbred mouse strains. Nature 448: $1050-1053$.

Goldstein DB. 2009. Common genetic variation and human traits. $N$ Engl J Med 360: $1696-1698$.

Grubb SC, Maddatu TP, Bult CJ, Bogue MA. 2009. Mouse phenome database. Nucleic Acids Res 37: D720-D730.

Guan Y, Myers CL, Lu R, Lemischka IR, Bult CJ, Troyanskaya OG. 2008. A genomewide functional network for the laboratory mouse. PLoS Comput Biol 4: e1000165. doi: 10.1371/journal.pcbi. 1000165.

Guttman M, Amit I, Garber M, French C, Lin MF, Feldser D, Huarte M, Zuk O, Carey BW, Cassady JP, et al. 2009. Chromatin signature reveals over a thousand highly conserved large non-coding RNAs in mammals. Nature 458: 223-227.

Hanlon P, Lorenz WA, Shao Z, Harper JM, Galecki AT, Miller RA, Burke DT. 2006. Three-locus and four-locus QTL interactions influence mouse insulin-like growth factor-I. Physiol Genomics 26: 46-54.

Hardy J, Singleton A. 2009. Genomewide association studies and human disease. N Engl J Med 360: 1759-1768.

Harper JM, Galecki AT, Burke DT, Pinkosky SL, Miller RA. 2003. Quantitative trait loci for insulin-like growth factor-I, leptin, thyroxine, and corticosterone in genetically heterogeneous mice. Physiol Genomics 15: $44-51$.

Hindorff LA, Junkins HA, Hall PN, Mehta JP, Manolio TA. 2011. A catalog of published genome-wide association studies. http://www.genome.gov/ gwastudies.

Jackson AU, Fornés A, Galecki A, Miller RA, Burke DT. 1999. Multiple-trait quantitative trait loci analysis using a large mouse sibship. Genetics 151: 785-795.

Jepsen KJ, Hu B, Tommasini SM, Courtland HW, Price C, Cordova M, Nadeau JH. 2009. Phenotypic integration of skeletal traits during growth buffers genetic variants affecting the slenderness of femora in inbred mouse strains. Mamm Genome 20: 21-33.

Kenney-Hunt JP, Wang B, Norgard EA, Fawcett G, Falk D, Pletscher LS, Jarvis JP, Roseman C, Wolf J, Cheverud JM. 2008. Pleiotropic patterns of quantitative trait loci for 70 murine skeletal traits. Genetics 178: 22752288.

Kraft P. 2008. Curses-winner's and otherwise-in genetic epidemiology. Epidemiology 19: 649-651.

Ku CS, Loy EY, Pawitan Y, Chia KS. 2010. The pursuit of genome-wide association studies: Where are we now? J Hum Genet 55: 195-206.

Kuhn JL, Goldstein SA, Feldkamp LA, Goulet RW, Jesion G. 1990. Evaluation of a microcomputed tomography system to study trabecular bone structure. J Orthop Res 8: 833-842.

Lipman R, Galecki A, Burke DT, Miller RA. 2004. Genetic loci that influence cause of death in a heterogeneous mouse stock. J Gerontol Biol Sci Med Sci 59: $977-983$. 
Lohmueller KE, Pearce CL, Pike M, Lander ES, Hirschhorn JN. 2003. Meta-analysis of genetic association studies supports a contribution of common variants to susceptibility to common disease. Nat Genet 33: 177-182.

McCarthy MI, Abecasis GR, Cardon LR, Goldstein DB, Little J, Ioannidis JPA, Hirschhorn JN. 2008. Genome-wide association studies for complex traits: Consensus, uncertainty and challenges. Nat Rev Genet 9: 356-369.

Miller RA, Harper JM, Galecki A, Burke DT. 2002. Big mice die young: Early life body weight predicts longevity in genetically heterogeneous mice. Aging Cell 1: 22-29.

Nadon NL. 2006. Aged rodents for biogerontology research. In Handbook of models for human aging (ed. PM Conn), pp. 393-401. Elsevier, New York.

Nicolae DL, Gamazon E, Zhang W, Duan S, Dolan ME, Cox NJ. 2010. Traitassociated SNPs are more likely to be eQTLs: Annotation to enhance discovery from GWAS. PLoS Genet 6: e1000888. doi: 10.1371/ journal.pgen.1000888.

Paigen K. 2003. One hundred years of mouse genetics: An intellectual history. II. The molecular revolution (1981-2002). Genetics 163: 1227-1235.

Peters LL, Robledo RF, Bult CJ, Churchill GA, Paigen BJ, Svenson KL. 2007. The mouse as a model for human biology: A resource guide for complex trait analysis. Nat Rev Genet 8: 58-69.

Phelan JP, Austad SN. 1994. Selecting animal models of human aging: Inbred strains often exhibit less biological uniformity than $\mathrm{F}_{1}$ hybrids. J Gerontol 49: B1-B11.

Quimby FW, Davisson MT, Fox JG, Newcomer CE, Barthold SW, ed. 2006. The mouse in biomedical research. Academic Press, New York.

Reed DR, Bachmanov AA, Tordoff MG. 2007. Forty mouse strain survey of body composition. Physiol Behav 91: 593-600.

Reeves GM, McCreadie BR, Chen S, Galecki AT, Burke DT, Miller RA, Goldstein SA. 2007. Quantitative trait loci modulate vertebral morphology and mechanical properties in a population of 18 month old genetically heterogeneous mice. Bone 40: 433-443.

Rosenthal N, Brown S. 2007. The mouse ascending: Perspectives for humandisease models. Nat Cell Biol 9: 993-999.

Soranzo N, Rivadeneira F, Chinappen-Horsley U, Malkina I, Richards JB, Hammond N, Stolk L, Nica A, Inouye M, Hofman A, et al. 2009. Meta-analysis of genome-wide scans for human adult stature in humans identifies novel loci and associations with measures of skeletal frame size. PLoS Genet 5: e1000445. doi: 10.1371/journal.pgen. 1000445.

Stranger BE, Stahl EA, Towfique RT. 2011. Progress and promise of genomewide association studies for human complex trait genetics. Genetics 187: 367-383.

Takada T, Mita A, Maeno A, Sakai T, Shitara H, Kikkawa Y, Moriwaki K, Yonekawa H, Shiroishi T. 2008. Mouse inter-subspecific consomic strains for genetic dissection of quantitative complex traits. Genome Res 18: $500-508$.

Valdar W, Solberg LC, Gauguier D, Cookson WO, Rawlins JN, Mott R, Flint J. 2006a. Genetic and environmental effects on complex traits in mice. Genetics 174: 959-984.

Valdar W, Solberg LC, Gauguier D, Burnett S, Klenerman P, Cookson WO, Taylor MS, Rawlins JN, Mott R, Flint J. 2006b. Genome-wide genetic association of complex traits in heterogeneous stock mice. Nat Genet $\mathbf{3 8}$ : 879-887.

Volkman SK, Galecki AT, Burke DT, Paczas MR, Moalli MR, Miller RA, Goldstein SA. 2003. Quantitative trait loci for femoral size and shape in a genetically heterogeneous mouse population. J Bone Miner 18: 1497-1505.

Volkman SK, Galecki AT, Burke DT, Miller RA, Goldstein SA. 2004. Quantitative trait loci that modulate femoral mechanical properties in a genetically heterogeneous mouse population. J Bone Miner 19: 1497-1505.

Wagner GP, Kenney-Hunt JP, Pavlicev M, Peck JR, Waxman D, Cheverud JM. 2008. Pleiotropic scaling of gene effects and the 'cost of complexity.' Nature 452: $470-472$.

Weiss KM. 2008. Tilting at Quixotic Trait Loci (QTL): An evolutionary perspective on genetic causation. Genetics 179: 1741-1756.

Yang J, Benyamin B, McEvoy BP, Gordon S, Henders AK, Nyholt DR, Madden PA, Heath AC, Martin NG, Montgomery GW, et al. 2010. Common SNPs explain a large proportion of the heritability for human height. Nat Genet 42: 565-569.

Received November 30, 2011; accepted in revised form April 2, 2012. 


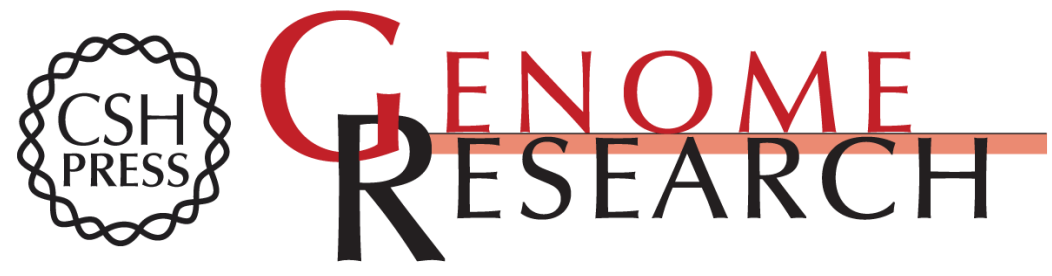

\section{Dissection of complex adult traits in a mouse synthetic population}

David T. Burke, Kenneth M. Kozloff, Shu Chen, et al.

Genome Res. 2012 22: 1549-1557 originally published online May 15, 2012

Access the most recent version at doi:10.1101/gr.135582.111

$\begin{array}{ll}\text { References } & \begin{array}{l}\text { This article cites } 46 \text { articles, } 2 \text { of which can be accessed free at: } \\ \text { http://genome.cshlp.org/content/22/8/1549.full.html\#ref-list-1 }\end{array}\end{array}$

Creative This article is distributed exclusively by Cold Spring Harbor Laboratory Press for the Commons

License

first six months after the full-issue publication date (see

http://genome.cshlp.org/site/misc/terms.xhtml). After six months, it is available under a Creative Commons License (Attribution-NonCommercial 3.0 Unported License), as described at http://creativecommons.org/licenses/by-nc/3.0/.

Email Alerting Receive free email alerts when new articles cite this article - sign up in the box at the Service top right corner of the article or click here.

\section{Affordable, Accurate Sequencing.}

To subscribe to Genome Research go to:

https://genome.cshlp.org/subscriptions 